\title{
Hazard function theory for nonstationary natural hazards
}

\author{
Laura K. Read and Richard M. Vogel \\ Department of Civil and Environmental Engineering, Tufts University, 200 College Avenue, Medford, MA, 02155, USA
}

Correspondence to: Laura K. Read (laurareads@gmail.com)

Received: 5 October 2015 - Published in Nat. Hazards Earth Syst. Sci. Discuss.: 13 November 2015

Revised: 17 March 2016 - Accepted: 18 March 2016 - Published: 11 April 2016

\begin{abstract}
Impact from natural hazards is a shared global problem that causes tremendous loss of life and property, economic cost, and damage to the environment. Increasingly, many natural processes show evidence of nonstationary behavior including wind speeds, landslides, wildfires, precipitation, streamflow, sea levels, and earthquakes. Traditional probabilistic analysis of natural hazards based on peaks over threshold (POT) generally assumes stationarity in the magnitudes and arrivals of events, i.e., that the probability of exceedance of some critical event is constant through time. Given increasing evidence of trends in natural hazards, new methods are needed to characterize their probabilistic behavior. The well-developed field of hazard function analysis (HFA) is ideally suited to this problem because its primary goal is to describe changes in the exceedance probability of an event over time. HFA is widely used in medicine, manufacturing, actuarial statistics, reliability engineering, economics, and elsewhere. HFA provides a rich theory to relate the natural hazard event series $(X)$ with its failure time series $(T)$, enabling computation of corresponding average return periods, risk, and reliabilities associated with nonstationary event series. This work investigates the suitability of HFA to characterize nonstationary natural hazards whose POT magnitudes are assumed to follow the widely applied generalized Pareto model. We derive the hazard function for this case and demonstrate how metrics such as reliability and average return period are impacted by nonstationarity and discuss the implications for planning and design. Our theoretical analysis linking hazard random variable $X$ with corresponding failure time series $T$ should have application to a wide class of natural hazards with opportunities for future extensions.
\end{abstract}

\section{Introduction}

Studies from the natural hazards literature indicate that certain processes show evidence of nonstationary behavior through trends in magnitudes over time. Such trends have been attributed to changes in climate patterns, e.g., for wind speeds (de Winter et al., 2013), wildfires (Liu et al., 2010), typhoons (Kim et al., 2015), and extreme precipitation (Roth et al., 2014), and also linked directly to human activities, e.g., increase in earthquakes from wastewater injection associated with hydraulic fracturing (Ellsworth, 2013). Other natural hazards such as floods resulting from streamflow (Di Baldassarre et al., 2010; Vogel et al., 2011) and from sea level rise (Obeysekera and Park, 2012) may be a result of a myriad of anthropogenic influences including climate change, land use change, and even natural processes such as land subsidence. In addition to evidence of magnitude changes of many natural hazards, recent reports document a corresponding surge in human exposure to natural hazards (Blaikie et al., 2014), along with a 14-fold increase in economic damages due to natural disasters since 1950 (Guha-Sapir et al., 2004). Given evidence of trends and the consequent expected growth in devastating impacts from natural hazards across the world, new methods are needed to characterize their probabilistic behavior and communicate event likelihood and the risk of failure associated with infrastructure designed to protect society against such events. The existing rich and evolving field of hazard function analysis (HFA) is well suited to problems in which the probability of an event is changing over time, yet to our knowledge has not been applied to natural hazards.

Probabilistic analysis of natural hazards normally takes one of two approaches in fitting a probability distribution to hazard event data series. As we summarize in Table 1, one common approach employs the peaks over threshold (POT) data set, also commonly referred to as the partial duration se- 
Table 1. Summary of natural hazards employing Poisson-GP model.

\begin{tabular}{|c|c|c|c|}
\hline Natural hazard & References & Evidence of nonstationarity in process & Nonstationary POT model formulated? \\
\hline Floods & $\begin{array}{l}\text { Todorovic (1978); } \\
\text { Madsen et al. (1997) }\end{array}$ & Bayazit (2015) for a review & $\begin{array}{l}\text { Yes, in Strupczewski et al. (2001); } \\
\text { Villarini et al. (2012) }\end{array}$ \\
\hline Earthquakes & $\begin{array}{l}\text { Gutenberg and Richter (1954); } \\
\text { Utsu (1999) }\end{array}$ & Ellsworth (2013) for a review & No \\
\hline Extreme rainfall & $\begin{array}{l}\text { Sugahara et al. (2009); } \\
\text { Bonnin et al. (2011) }\end{array}$ & $\begin{array}{l}\text { Begueria et al. (2011); Tramblay et al. (2013); } \\
\text { Roth et al. (2014); Sugahara et al. (2009) }\end{array}$ & Yes, in all references \\
\hline Wildfires & Holmes et al. (2008) & Liu et al. (2010) & No \\
\hline Extreme wind speeds & $\begin{array}{l}\text { Palutikof et al. (1999) for review; } \\
\text { Jagger and Elsner (2006) }\end{array}$ & $\begin{array}{l}\text { Young et al. (2011); } \\
\text { Pryor and Barthelmie (2010) for review }\end{array}$ & Young et al. (2011) \\
\hline $\begin{array}{l}\text { Wave height (proxy } \\
\text { for storm surge) }\end{array}$ & Davison and Smith (1990) & $\begin{array}{l}\text { Méndez et al. (2006); Young et al. (2011); } \\
\text { Ruggerio et al. (2010) }\end{array}$ & $\begin{array}{l}\text { Yes, in Méndez et al. (2006); } \\
\text { Ruggerio et al. (2010) }\end{array}$ \\
\hline $\begin{array}{l}\text { Daily max and } \\
\text { min temperatures }\end{array}$ & Waylen (1988) & Keellings and Waylen (2014) & Yes, but not derived in text \\
\hline Ecological extremes & Katz et al. (2005) & $\begin{array}{l}\text { Katz et al. (2005) } \\
\text { (sediment yield) }\end{array}$ & Yes \\
\hline
\end{tabular}

ries (PDS), to characterize exceedances above some defined magnitude (threshold) that occur over an interval of time. The second method is to fit a probability distribution to the annual maximum series (AMS), common practice in hydrology (Gumbel, 2012; Stedinger et al., 1993) and also appropriate for earthquakes (Thompson et al., 2007) and many other processes (Beirlant et al., 2006; Coles et al., 2001). Hydrologists have extensively studied the theoretical relations between POT and AMS methods (Stedinger et al., 1993; Todorovic, 1978) and compared the two for characterizing the probabilities of flood events (Madsen et al., 1997). In general the POT approach appears to provide a larger data set to draw from; however as the threshold of exceedance used to define the POT series is lowered, the series of maxima begin to exhibit temporal dependence which complicates the probabilistic analysis considerably. Further complexities arise in POT analyses due to subjectivity of the threshold and difficulty in confirmation of independence between events (Stedinger et al., 1993).

Still the POT method is widely used for many natural phenomena either because there is an intuitive choice for the threshold of exceedance, as in the case of earthquakes where the magnitude of completeness is often selected, or because the analyst wishes to maximize the use of data and does not always understand the tradeoff between the length of the POT series and the inherent increase in its temporal dependence structure. Due to the ubiquitous application of the POT method, a substantial number of textbooks and articles have studied methods for minimizing the difficulty of implementation, with emphasis on the subjectivity of threshold selection and evaluation of independence of events (Davison and Smith, 1990; Smith, 2003). This study assumes a POT approach is taken for characterizing the magnitudes of natural hazards, following many examples from the literature including extreme winds (Palutikof et al., 1999), earthquakes (Pisarenko and Sornette, 2003), wildfires (Schoenberg et al., 2003), and wave heights (Lopatoukhin et al., 2000), among others. Future work will extend these analyses to AMS series (Read, 2016).

\subsection{Application of general POT model in natural hazards}

The generalized Pareto (GP) distribution, a generalization of the exponential distribution, was first introduced as a limiting distribution for modeling high level exceedances by Pickands III (1975) and later developed by Hosking and Wallis (1987), who discuss its theory and an application for extreme floods. Davison and Smith (1990) provide techniques for dealing with serially dependent and seasonal data for modeling exceedances above a threshold with the GP distribution. Hosking and Wallis (1987) discuss the fundamental properties of the GP distribution. See Pickands III (1975) and Davison and Smith (1990) for further theoretical background on the application of the GP distribution for modeling POT series. In general, the GP distribution arises for variables whose distributions are heavy-tailed, in cases where the lighter-tailed exponential distribution does not provide sufficient robustness (Hosking and Wallis, 1987).

The GP distribution has been widely applied to natural hazards (see Table 1) and in many other fields including financial risk, insurance, and other environmental problems (Smith, 2003) to characterize the magnitude of exceedances above a threshold. Hosking and Wallis (1987), Stedinger et al. (1993), and others show that if the time between the peaks corresponding to a POT series follow a Poisson distribution, and the POT magnitudes follow an exponential distribution, then the AMS follow a Gumbel distribution. Similarly, Hosking and Wallis (1987) and others show that if time between the peaks of the POT series are Poisson and the POT magnitudes follow a two-parameter GP (GP2) distribution, then the AMS follows a generalized extreme value distribution. Table 1 lists many natural hazards problems that apply the Poisson-GP model for their probabilistic analysis. Here 
we consider a POT that follows the GP model (the exponential distribution is a special case of the GP model when the limit of the shape parameter approaches 0; see Davison and Smith (1990) for details). For example the GutenbergRichter model developed for earthquake magnitudes is a twoparameter exponential model (Gutenberg and Richter, 1954).

For the natural hazards listed in Table 1, the common approach is to assume that the probability of exceedance $(p)$ for a given magnitude event is constant from year to year, i.e., stationary through time. Under the assumption of stationarity, the theoretical relationships between POT and AMS enable straightforward computation of summary and design metrics such as the quantile or percentile of the distribution associated with a particular average return period and/or reliability (Stedinger et al., 1993). When evidence of nonstationarity, or a trend in either or both the frequency or the magnitude of the exceedance events occurring through time is detected, then $p$ can no longer be assumed as a constant and the traditional Poisson-GP (or other) model must be modified to account for dependence on time and/or some other explanatory co-variate. Not adjusting the probabilistic analysis for a positive trend when one is present can lead to gross overestimation of the expected return period and reliability of a system, as shown for floods by several recent studies (Salas and Obeysekera, 2014; Read and Vogel, 2015). Furthermore, Vogel et al. (2013) document that without a rigorous probabilistic analysis of trends in natural hazards, we may overlook and fail to prepare for a wide range of societal outcomes which may have occurred repeatedly in the past.

\subsection{Introduction to hazard function theory and implications for natural hazards}

Despite the similarity in name, the application of the theory of HFA, also commonly referred to as survival analysis, is practically absent from general literature in the field of natural hazards. HFA is a well-established set of tools useful for conducting a "time-to-event" analysis or for understanding the distribution of survival (failure) times for a given process (e.g., survival rate of a chronic disease, time until electrical burnout of a device, age-specific mortality rate). This is precisely the concern of those modeling natural hazards that are changing over time. Generally, HFA is comprised of three primary functions: (1) the hazard function, $h(t)$, which is defined as the failure rate, or as the likelihood of experiencing a failure at a particular point in time; (2) the survival function, $S_{T}(t)$, defined as the exceedance probability for the random variable time $(T)$ or in reliability engineering as the cumulative distribution function (CDF) of $T, F_{T}(t)$, where $S_{T}(t)=1-F_{T}(t)$; and (3) the cumulative hazard function, $H(t)$, interpreted as the total number of failure events over a period of time.

Most applications of HFA are interested in computing design metrics based on knowledge of $h(t), S_{T}(t)$, and $H(t)$, e.g., the mean time to failure, or the reliability of surviving a certain amount of time without at least one failure event. In nearly all of the literature on HFA, the process for defining these three functions begins in one of two ways: either by first identifying an appropriate hazard function $h(t)$, a possible path if sufficient knowledge (or empirical evidence) of the failure process is known (e.g., does the probability of failure increase or decrease, or is it constant over time); or by estimating the survival function $S_{T}(t)$ by fitting a set of survival time data to a distribution (Klein and Moeschberger, 1997). Neither of these two approaches are particularly suited to natural hazards, because until this paper there has not been any guidance on how to select an appropriate a hazard function $h(t)$ for a natural hazard random variable. While reliance on empirical data to fit a survival function to data is a common approach in other fields, often in regards to natural hazards our data set is incomplete.

The theory of HFA is derived elsewhere and summarized in numerous textbooks (Finkelstein, 2008; Kleinbaum and Klein, 1996; Klein and Moeschberger, 1997); hence we only summarize the relevant relationships among the hazard rate function $h(t)$, the probability density function (PDF) of the time to failure $f_{T}(t)$, the CDF of the time of failure $F_{T}(t)$, its corresponding survival function $S_{T}(t)$, as well as the cumulative hazard function $H(t)$ :

$$
\begin{aligned}
& h(t)=\frac{f_{T}(t)}{1-F_{T}(t)}, \\
& S_{T}(t)=1-F_{T}(t)=\exp \left(-\int_{0}^{t} h(s) \mathrm{d} s\right), \\
& H(t)=-\ln \left(S_{T}(t)\right)=\int_{0}^{t} h(s) \mathrm{d} s .
\end{aligned}
$$

HFA has been applied in the fields of bio-statistics and medicine (Cox, 1972; Pike, 1966) as well as many other disciplines including economics (Kiefer, 1988) and engineering (Finkelstein, 2008; Hillier and Lieberman, 1990). Very little attention has been given to the use of HFA to natural hazards (Read, 2016; Katz and Brown, 1992; Lee et al., 1986). Concepts from hazard function theory were applied to develop dynamic reliability models for characterizing composite risk of hydrologic and hydraulic failures in conveyance systems in the 1980s (Lansey, 1989; Tung, 1985; Tung and Mays, 1981).

The primary goal of this work is to use the theory of HFA to link the probabilistic properties of $T$ with properties of the probability distribution for a nonstationary natural hazard event $X$. We begin by explicitly relating $h(t)$ to exceedance probability associated with the event magnitudes for a general hazard, $X$, assuming the natural hazard POT follows a GP distribution. We then derive $S_{T}(t), H(t)$ and $\mathrm{MTTF}=E[T]$ for the case of the GP2 model. We test the assumption that $h(t)=p_{t}$ by comparing theoretical $S_{T}(t)$ to empirical survival time values derived from Monte Carlo ex- 
periments. Since an exponential model is a special case of the GP model, our results also apply to POT series which follow an exponential model. Recall that the exponential distribution is of interest when the AMS is Gumbel, which is applicable for many natural hazards. Since both the exponential and GP2 models are widely used for representing natural event magnitudes in a POT, the analysis presented here is relevant for a wide range of nonstationary natural hazards. We hope to demonstrate that HFA can be a useful methodology for characterizing nonstationary natural hazards, for communicating natural hazard event likelihood under nonstationarity, and for computing corresponding design metrics that reflect the changing behavior of the both the magnitude and frequency of a natural hazard through time.

\section{Hazard function analysis for nonstationary natural hazard magnitudes}

To relate the properties of $h(t)$ with the magnitudes of a particular natural hazard $(X)$, we first consider the stationary situation in which a natural hazard event has $\operatorname{CDF} F_{X}(x ; \theta)$, with generic parameter set $\theta$ that does not change in time, and the exceedance probability $p_{o}$ associated with some threshold magnitude value $x_{o}$ is constant through time. In the stationary case, the hazard failure rate, $h(t)$, is constant so that $h(t)=p_{o}$ (Read, 2016), and the time to failure, $T$, always follows an one-parameter exponential distribution (or if discrete, the geometric distribution); computation of the average return period is readily obtained from probability theory as the expected value of the exponential variable $E[T]=1 / p$.

If the magnitudes of a natural hazard exhibit an increasing trend through time, this indicates that the exceedance probability associated with a particular design event is changing with time, which we denote as $p_{t}$. In such a situation, the expectation $E[T]$ is no longer a sufficient statistic for the distribution of $T$, and a more complex analysis is needed (Read and Vogel, 2015).

For a nonstationary natural hazard, the parameters of the $\mathrm{CDF}$ of $X$ are no longer constant and the CDF is now given as $F_{X}(x ; \theta(t))$. For a process with increasing hazard event magnitudes, this implies that the exceedance probability is also increasing with time such that $p_{t}=1-F_{X}(x ; \theta(t))$. Likewise, $h(t)$ is no longer constant and, as we will show, can be computed directly from a probabilistic analysis of the natural hazard of interest by equating $h(t)=p_{t}$. This assumption is the primary difference between this study and other HFA applications in the literature, as we explicitly link $h(t)$ to the event magnitudes of a natural hazard rather than use empirical evidence (fitting) The advantage of this approach is to generalize across many types of natural hazards such that future researchers can pursue HFA as an alternate methodology for describing the probabilistic behavior of nonstationary natural hazards.

\section{GP2 model for magnitudes of natural hazards}

As discussed earlier, the GP distribution is widely used in modeling the magnitudes above a pre-defined threshold for a variety of natural hazards. In this section we present the GP2 stationary and nonstationary models, reviewing literature to support the selection of our nonstationary natural hazard model formulation. We then use HFA theory to derive $h(t), S_{T}(t)$, and $H(t)$ for the GP2 nonstationary model and discuss the findings and interpretations for each function.

\subsection{Stationary GP2 model}

We begin by describing the definitions for a random hazard variable $X$ whose POT event magnitudes follow a GP2 distribution. The stationary PDF and CDF, introduced by Hosking and Wallis (1987), are

$f_{x}(x)=\frac{1}{\alpha}\left[1-\kappa\left(\frac{x}{\alpha}\right)\right]^{\frac{1}{\kappa}-1}$ for $\kappa \neq 0$,
$F_{x}(x)=1-\left[1-\kappa\left(\frac{x}{\alpha}\right)\right]^{\frac{1}{\kappa}}$ for $\kappa \neq 0$,

where $\alpha$ is the scale parameter and $\kappa$ is the shape parameter. We use the coefficient of variation, $C_{x}=\sigma_{x} / \mu_{x}$, to represent the variability of the system. Note that when $\kappa=0$ and $C_{x}=1$, Eqs. (4) and (5) reduce to the exponential distribution with a mean of $\alpha$; this form corresponds to a Gumbel distribution for the AMS. The first and second moments of $X$ are

$\mu_{x}=\frac{\alpha}{1+\kappa}$,

$\sigma_{x}^{2}=\frac{\alpha^{2}}{(1+\kappa)^{2}(1+2 \kappa)}$.

Combining Eqs. (6) and (7) for the GP2 model yields

$C_{x}=\frac{1}{\sqrt{2 \kappa+1}}$.

The quantile function for the GP2 distribution for a design event, $x_{o}$, associated with exceedance probability, $p$, is written by rearranging Eq. (5) as

$x_{o}=\frac{\alpha}{\kappa}\left[1-p^{\kappa}\right]$.

These equations serve as the foundation for developing a nonstationary GP2 model, discussed in the next section.

\subsection{Nonstationary GP2 model}

Although we could not locate any previous research mathematically linking HFA and nonstationary natural hazards, there are numerous papers that employ a nonstationary GP model for the POT magnitudes of specific natural hazards (shown in Table 1). We briefly review those models to provide context for the trend model adopted here. Literature on 
nonstationary GP models for specific natural hazards has employed a variety of parameterizations. For example, Roth et al. $(2012,2014)$ considered models of changes in the POT threshold over time and Strupczewski et al. (2001) modeled the arrival time distribution of the POT with time-varying Poisson parameters. Strupczewski et al. (2001) also modeled the changes in the magnitudes of the POT events over time by modeling changes in the GP parameters over time as we do here.

Nearly all previous studies that employed nonstationary POT models in the context of natural hazards adopt some form of the Poisson-GP model, and many whose concerns regard increasing magnitudes have been specific to extreme rainfall. With respect to extreme daily rainfall, most have built nonstationary GP2 models assuming a trend in the scale parameter $(\alpha)$, either modeled linearly (Beguería et al., 2011; Sugahara et al., 2009) or log-linearly (Tramblay et al., 2013). Roth et al. (2014) note that modeling a trend in the threshold level itself indicates a comparable trend in the scale parameter. Tramblay et al. (2013) used time-varying co-variates in the Poisson arrivals (occurrence of seasonal oscillation patterns) and in the magnitudes (monthly air temperature) to model heavy rainfall in southern France and found improvement from the stationary model. As pointed out by Khaliq et al. (2006), Tramblay et al. (2013), and others, it is less common to vary the shape parameter $(\kappa)$ through time due to difficulty with precision and a lack of evidence on model improvement with a time-varying shape parameter.

Studies from other natural hazards are consistent with those in extreme rainfall for nonstationary Poisson-GP model formulations, though with more examples of time variation in the shape parameter. For example, Strupczewski et al. (2001) used linear and parabolic trends in both $\alpha$ and $\kappa$ to model flood magnitudes; others have explored linear models in $\kappa$ for extreme winds (Young et al., 2011) and in sediment yield (Katz et al., 2005). For wave height, several assumed a trend in the location parameter either as linear (Ruggiero et al., 2010) or log-linear (Méndez et al., 2006) formulations. Renard et al. (2006) used a Bayesian approach to explore step change and linear trend models in $\alpha$ for general purpose with an application to floods. The Bayesian framework was also used by Fawcett and Walshaw (2015) to present a new hybridized method for estimating more precise return levels for nonstationary storm surge and wind speeds.

\subsection{Derivation of nonstationary GP2 hazard model}

Our approach is to derive the primary HFA functions $f_{T}(t)$, $F_{T}(t), S_{T}(t), h(t)$, and $H(t)$ using the nonstationary CDF, $F_{X}(x ; \theta(t))$, for a GP2 random variable $X$. To develop a nonstationary GP model, we employ an exponential trend model in the scale parameter $\alpha_{x}(t)$, so that

$\alpha_{x}(t)=\alpha_{o} \exp (\beta t)$
The model in Eq. (10) is equivalent to a model of the conditional mean of the natural hazard $X$ and has been found to provide an excellent representation of changes in the mean annual flood for flood series at thousands of rivers in the United States (Vogel et al., 2011) and in the United Kingdom (Prosdocimi et al., 2014). This model is described by Khaliq et al. (2006) and was also used in Tramblay et al. (2013) for extreme rainfall.

We assume that the shape parameter $\kappa$ is constant through time as consistent with previous studies discussed earlier. This assumption implies that $C_{x}$ is fixed (Eq. 10), or that the variability of the system is assumed constant over the time period, defined at $t=0$, and thus the standard deviation changes in step with the mean (parameterized by $\alpha$ ). Again, there is reasonable evidence that this is the case for floods (see Vogel et al., 2011; Prosdocimi et al., 2014).

Following Vogel et al. (2011), Prosdocimi et al. (2014), and Read and Vogel (2015), we replace the trend coefficient $\beta$ in Eq. (10) with the more physically meaningful magnification factor $M$ to represent the ratio of the magnitude of the natural hazard quantile at time period $(t+d)$ to the natural hazard quantile at time $t$. For the model developed here, the magnification factor, $M$, can be derived by combining the GP2 quantile function in Eq. (9) and the trend model in Eq. (10), inserting into the expression below:

$M=\frac{x_{o}(t+d)}{x_{o}(t)}=\frac{\frac{1}{\lambda} \exp \left[\beta(t+d) \ln \left(p_{t}\right)\right]}{\frac{1}{\lambda} \exp \left[\beta t \ln \left(p_{t}\right)\right]}=\exp [\beta \cdot d]$.

Thus $M$ reflects the change in the magnitude of the natural hazard over time. So for example, $M=2$ and $d=10$ indicates that the magnitudes of the natural hazard have increased twofold over $d$, for all values of $p$.

In this section we derive $h(t), S_{T}(t), H(t)$, and $f_{T}(t)$ for the nonstationary GP2 model. First recall that the hazard function is equal to the exceedance probability through time for a natural hazard event series, $h(t)=p_{t}$. Using the relationships above we derive an expression for $h(t)$ dependent only on fundamental parameters $M, p_{o}$, and $C_{x}$.

Consider that in Eq. (9) the design event is fixed as $x_{o}$ and associated with $p_{o}$ and $\alpha_{o}$ at time $t=0$. To derive the expression of interest, $h(t)=p_{t}=1-F_{X}\left(x_{o} ; \theta(t)\right)$, we insert the quantile function in Eq. (9) and trend model in Eq. (10) into Eq. (5), replacing $\beta$ with $M$ by combining with Eq. (11). The result yields the hazard function:

$h(t)=p_{t}=1-F_{C}\left(x_{o} ; t\right)=\left[1-\frac{\left(1-p_{o} \frac{1-C_{x}^{2}}{2 C_{x}^{2}}\right)}{M^{t / d}}\right]^{\frac{2 C_{x}^{2}}{1-C} x}$,

where $\kappa$ is replaced with $C_{x}$ after rearranging Eq. (8) and $d$ is the time step associated with $M$. Combining the theoretical relationships in Eqs. (1)-(3) with Eq. (12) leads to expressions for $S_{T}(t), H(t)$, and $f_{T}(t)$ solved by numeric integration. 


$$
\begin{aligned}
& S_{T}(t)=\exp \left(-\int_{0}^{t} b \mathrm{~d} s\right) \\
& H(t)=\int_{0}^{t} b \mathrm{~d} s .
\end{aligned}
$$

Finally, the PDF of the time to failure distribution for the GP2 nonstationary model is

$$
f_{T}(t)=b \cdot \exp \left(-\int_{0}^{t} b \mathrm{~d} s\right)
$$

where $b=\left[\frac{1-\left(1-p^{\frac{1-C_{x}^{2}}{2 C_{x}^{2}}}\right)}{M^{s / d}}\right]^{\frac{2 C_{x}^{2}}{1-C_{x}^{2}}}$.

For the case of the one-parameter exponential, these functions simplify to $h(t)=p_{o}^{M^{-t / d}}, \quad S_{T}(t)=$ $\exp \left[-\int_{0}^{t} p_{o}^{M^{-s / d} \mathrm{~d} s}\right], H_{T}(t)=\int_{0}^{t} p_{o}^{M^{-s / d} \mathrm{~d} s}$, and $f_{T}(t)=\frac{d}{\mathrm{~d} t}$ $\left[\exp \left(\int_{0}^{t} p_{o}^{M^{-s / d} \mathrm{~d} s}\right)\right]$ (see Read (2016) for a complete derivation).

\subsection{Impacts of nonstationarity on probabilistic analysis of natural hazards using HFA}

In this section we explore how HFA can characterize the behavior of nonstationary natural hazards whose PDS magnitudes follow a GP2 model. Our results are exact (within the limitations of numerical integration) because they result from the derived analytical equations in Eqs. (12)-(15) for the HFA functions $f_{T}(t), F_{T}(t)$, and $S_{T}(t), h(t)$, and $H(t)$ corresponding to a natural hazard $X$ which follows a GP2 model. With no loss in generality, we assume the mean of the GP2 natural hazard of unity. We investigate the impact of small and large trends (corresponding to magnification factors, $M$, ranging from 1 to 1.25 with $d=10$ ) for a range of physical systems characterized by a range in variability corresponding to a range in the coefficient of variation of $X, C_{x}$, from 0.5 to 1.5 corresponding to a range in the GP2 shape $\kappa$ between -0.28 to 1.5$)$ for three event sizes $\left(p_{o}=0.01,0.002\right.$, $0.001)$. We verified our assumption that $h(t)=p_{t}$ for the GP2 case presented here by simulating failure times from a GP2 random variable for a range of $p_{o}, M$, and $C_{x}$ values and comparing to the theoretical $S_{T}(t)$ curves.

Figure 1 presents the hazard function $h(t)$, examining how a particular design event $p_{o}=0.002$ (500-year event) is influenced by the magnitude of the trend and system variability: (a) increasing variability, $C_{x}=0.75,1.25,1.5$ for a
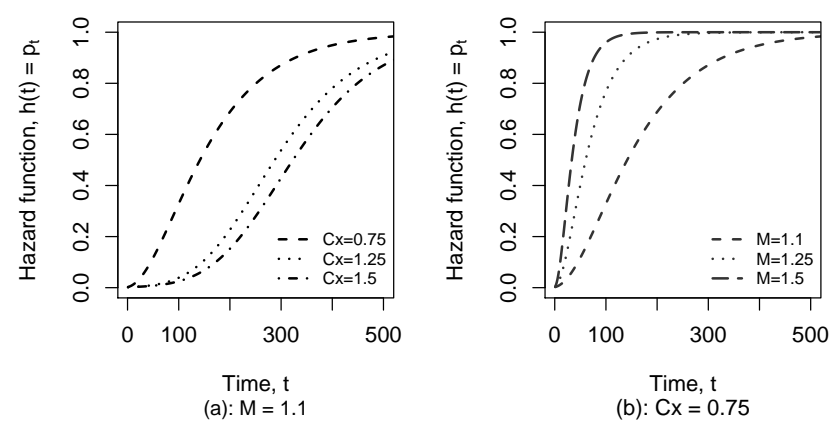

Figure 1. Hazard function $h(t)$ for the nonstationary GP2 model and $p_{o}=0.002$ for (a) a range of variability $\left(C_{x}=0.75,1.25,1.5\right)$, given $M=1.1$, and (b) a range of trend values $(M=1.1,1.25,1.5)$, given $C_{X}=0.75$.

set $M=1.1$; and (b) increasing trend values, $M=1.1,1.25$, 1.5 for a set $C_{x}=0.75$. Note that in the stationary case, $p_{t}=p_{o}=0.002$ results in a constant horizontal line, and as the magnitude of the trends increases (as $M$ increases), the hazard rate $h(t)$ tends toward unity earlier in time. Even from this initial relatively simplistic investigation we find that the hazard functions exhibit complex shapes, with some exhibiting inflection points and others without an inflection. Another important point is that the variability of the hazard magnitudes (characterized by the shape of the PDF of $X$ ) impacts the rate at which $h(t)$ increases, so that less variable hazards tend to have higher hazard rates than more variable hazards. This point is perhaps initially counter-intuitive, and our interpretation is that if a hazard is more consistent (with less variability), a larger trend ensures exceedance more so than a less consistent system that has a wider range of small and large events. This finding is relevant for planning purposes as it indicates which systems may be greater impacted by nonstationarity.

Typically in HFA work, the survival function $S_{T}(t)$ is presented as a primary figure in understanding risk of failure and likelihood of experiencing an exceedance event within a given period of time. Since $S_{T}(t)$ also represents the relationship between system reliability and time and because many fields employ the concept of reliability to protect against natural hazards, the $S_{T}(t)$ function is also relevant for planning purposes in this context (see Read and Vogel (2015) for further discussions relating to flood management and design). Figure 2 illustrates $S_{T}(t)$ for a $p_{o}=0.002$ event with a fixed $C_{x}=0.75$ representing a slightly less variable system and a range of increasing trends $(M=1.02,1.1,1.25)$ compared with stationary conditions $(M=1)$. Points represent simulated failure times using Monte Carlo analysis and lines are theoretical $S_{T}(t)$ from Eq. (13). The agreement between the simulations and theoretical lines verifies the assumption that $h(t)=p_{t}$. Additionally, Fig. 2 shows that even a small trend significantly reduces the system reliability compared with our expectations under stationary conditions. For example, 


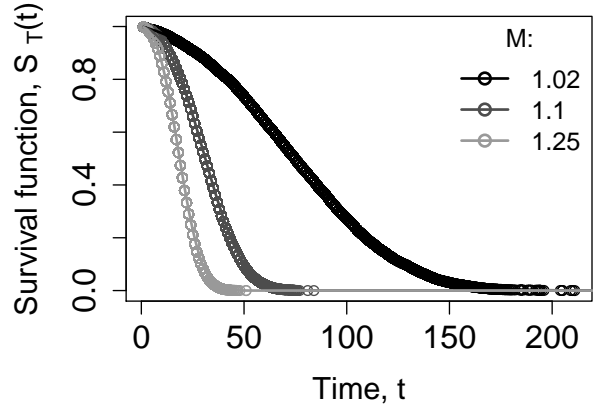

Figure 2. Survival function $S_{T}(t)$ for the nonstationary GP2 model, $p_{o}=0.002$ and $C_{x}=0.75$, for a range of trend values $(M=1,1.02$, 1.1, 1.25); lines represent theory (Eq. 13) and points are empirical values from Monte Carlo simulation.

the reliability of a structure designed to protect against a 500year event under stationary conditions after 50 time periods is quite high $\left(S_{T}(t)=0.90\right)$; however, as $M$ increases, the reliability decreases significantly, approaching 0 for $M=1.1$ and 1.25 at $t=50$. This suggests that if one was designing infrastructure to withstand a particularly large magnitude event over a planning period, under nonstationary conditions, the design would need to be significantly larger, and it may not even be possible to design a structure to achieve the same reliability as expected under stationary conditions.

A unique tool offered by HFA that can provide advancements in planning for nonstationary natural hazards is the cumulative hazard function $H(t)$, which represents the total hazard over a given amount of time (Wienke, 2010). For example, if $p_{o}=0.002$, as expected under stationary conditions, $H(t)=1$ for $t=500$, or we will experience, on average, one exceedance event every 500 years. However, if a trend with a magnification factor $M=1.1(d=10)$ is introduced in the same system, the time it takes for $H(t)=1$ is about 36 time periods, or another view, $H(t)=333$ events for $t=500$. Figure 3 illustrates these interpretations for two exceedance event sizes, fixed $C_{x}=0.75$ : (a) $p_{o}=0.002$, showing the number of time periods until $H(t)=1$; and (b) $p_{o}=0.001$, showing the total number of events over time. In Fig. 3a, we note that the stationary $M=1$ line corresponds with the $H(t)=1$ for $t=500$ as expected, and that as $M$ increases, the time until an exceedance event occurs dramatically decreases (note the $\log x$ axis scale). Figure $3 \mathrm{~b}$ depicts a similar story but illustrates an alternate interpretation: the total number of exceedance events over a time period for the rarer $p_{o}=0.001$ event, where $H(t)$ ranges from 1 for $t=1000$ as expected under stationary conditions to $H(t)=10+$ events in fewer than 50 time periods with a large $M$.

When one wishes to communicate the risk of failure and event likelihood, the cumulative hazard function is a useful metric for describing total risk (or reliability) over a certain planning horizon. While our analysis assumes that the trend
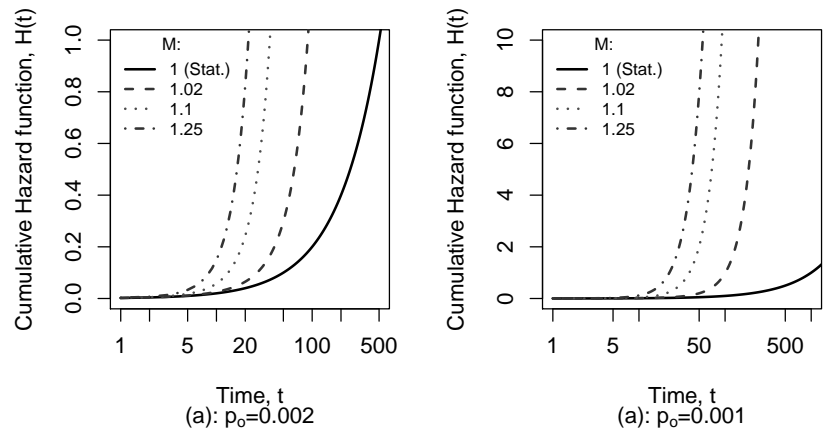

Figure 3. Cumulative hazard function $H(t)$ for the nonstationary GP2 model, with a fixed $C_{x}=0.75$ for a range of trend values ( $M=1,1.02,1.1,1.25)$; panels show two different size exceedance events: (a) $p_{o}=0.002$ and (b) $p_{o}=0.001$.
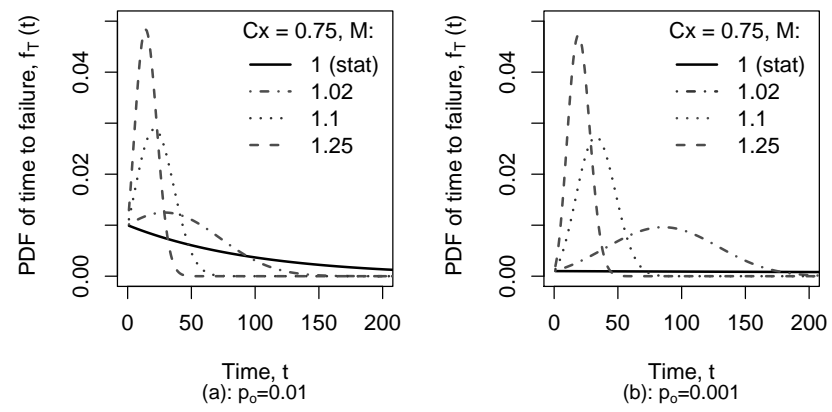

Figure 4. PDF of the time to failure distribution for the nonstationary GP2 model, with a fixed $C_{x}=0.75$ for a range of trend values $(M=1,1.02,1.1,1.25)$; panels show three exceedance event sizes increasing in extremity: (a) $p_{o}=0.01$ and (b) $p_{o}=0.001$.

would increase over the entire time period, perhaps a "worst case" scenario, our results show that in the presence of an increasing trend in the POT series, we may experience far more exceedance events than expected under stationary conditions. Ignoring such trends may result in significant increased damages and losses from under-design of infrastructure or insufficient planning in populated areas.

After computing $S_{T}(t)$ and $H(t)$ we can easily use Eq. (1) to determine the PDF of the time to failure distribution for the nonstationary GP2 model by Eq. (15). Since we are interested in the behavior of $f_{T}(t)$ due to trends on a range of physical systems and for extreme events, we plot $f_{T}(t)$ for a fixed $C_{x}=0.75$ in Fig. 4, for a range of increasing trends $(M=1,1.02,1.1,1.25)$ and two event sizes $\left(p_{o}=\right.$ (a) 0.01 and (b) 0.001). We note that the shape of $f_{T}(t)$ evolves from the expected exponential curve under stationary conditions, to a more symmetric or normally distributed shape as $M$ increases. These results complement those by Read and Vogel (2015) who show similar behavior for a nonstationary two-parameter lognormal model of an AMS series of floods.

Similarly, in Fig. 5 we fix the trend at $M=1.05$ and explore the behavior of $f_{T}(t)$ over a realistic range of $C_{x}$ values 
$(0.5,0.75,1.5)$, for the same two event sizes $\left(p_{o}=\right.$ (a) 0.01 and (b) 0.001). As consistent with Fig. 1 showing $h(t)$ for various $C_{x}$ values, the shape of $f_{T}(t)$ in less variable systems (lower $C_{x}$ ) is more impacted by a trend than a more variable system, as indicated by the sharp peaks and shift in timing of the peaks (Fig. 5).

Our investigation of the behavior of $f_{T}(t)$ for the nonstationary GP2 model indicates that the shape and timing of the distribution changes with both the magnitude of the trend and variability of natural hazard. We also note that $f_{T}(t)$ exhibits complex patterns under nonstationary conditions, e.g., $f_{T}(t)$ is less impacted in shape/timing by $M$ for smaller events $\left(p_{o}\right)$, and that the presence of a trend leads to a range of shapes (approaching normal for large positive $M$ ) of the time to failure distribution. This more complicated behavior implies that under the premise of nonstationarity, we can no longer assume the failure time distribution is exponential in shape and that the MTTF is equal to $1 / p$. In fact, the mean of the distribution of $T$ is no longer a sufficient statistic as is the case under stationary conditions. We are the first to document such changes in the context of natural hazards for the GP2 distribution and anticipate that others will continue to do so for other events and distributions that exhibit nonstationarity. Using the derivations of $h(t), S_{T}(t), H(t)$, and $f_{T}(t)$ that we have provided here, one can use knowledge of the system $\left(M, C_{x}\right)$ and existing design metrics $\left(p_{o}\right.$ and reliability standards) in combination with HFA to better understand and characterize natural hazards as they change through time.

\section{Summary and conclusions}

We have presented a general introduction to the probabilistic analysis of nonstationary natural hazards using the welldeveloped field of HFA. We cite numerous sources of evidence which suggests that the magnitudes of many natural hazards are increasing, thus requiring new tools for conducting frequency analysis. To the authors' knowledge, the analysis and discussion presented here provides the first formal probabilistic analysis that draws a mathematical linkage between the magnitude of a natural hazard event $(X)$ with the distribution of failure times $(T)$ for the occurrence of some event exceeding a design threshold $\left(x_{o}\right)$. Through the lens of HFA, we have presented an alternative methodology for characterizing the likelihood of a natural hazard whose event magnitudes follow a GP2 distribution and are increasing over time. By explicitly linking properties of the time to failure $T$ with the exceedance probability $p$ of a natural hazard $(X)$, we have derived the primary hazard analysis equations: the hazard function $h(t)$, the survival (reliability) function $S_{T}(t)$, the cumulative hazard function $H(t)$, and the PDF of the time to failure distribution $f_{T}(t)$ corresponding to a POT series of natural hazards which follow the GP2 distribution. We demonstrate that the assumption $h(t)=p_{t}$ is valid for the
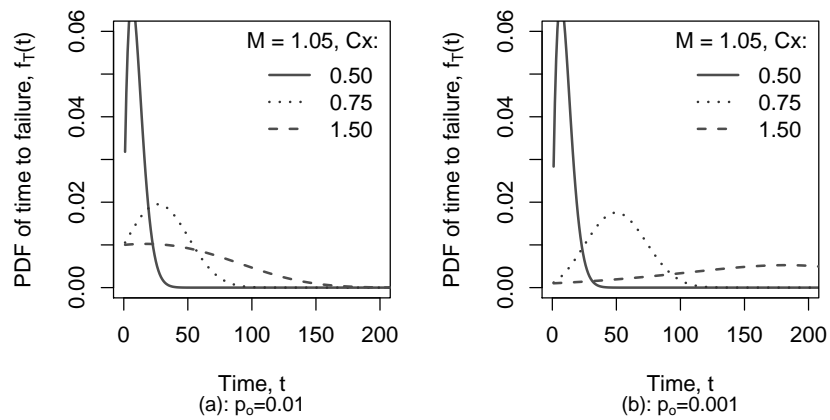

Figure 5. PDF of the time to failure distribution for the nonstationary GP2 model, with a fixed $M=1.05$ for a range of trend values $\left(C_{x}=0.5,0.75,1.50\right)$; panels show three exceedance event sizes increasing in extremity: (a) $p_{o}=0.01$ and (b) $p_{o}=0.001$.

case presented by comparing Monte Carlo simulated failure times from a GP2 random variable with $F_{X}\left(x_{o} ; \theta(t)\right)$ with HFA theory. We parameterize this GP2 model such that it only depends on the design exceedance probability at time $t=0, p_{o}$, the known system variability $C_{x}$, and the magnification factor $M$, and we use this to explore the impact of positive trends on the reliability or survival $S_{T}(t)$ until an exceedance event.

Findings of this investigation suggest that under nonstationary conditions, medium and large events could occur with much greater frequency than under stationary conditions (no trend). We find that the total number of hazards as characterized by $H(t)$ within a given planning period may substantially increase in the presence of a positive trend in the POT magnitudes of a natural hazard. Perhaps most importantly, under nonstationary conditions, the distribution of the time to failure is no longer exponentially distributed, and instead takes on a distribution with different shapes, depending on the variability of the hazard and the magnitude of the trend. As trend magnitudes increase for a range of event sizes $\left(p_{o}\right)$, the shape of the distribution of the survival time approaches normality and exhibits a sharp peak with a heavy upper tail. We also find that variability impacts the shape and timing of this peak in $f_{T}(t)$, such that less variable systems (lower $C_{x}$ ) are more affected by larger $M$ values, i.e., produce a more pronounced peak and a greater shift in timing.

The implications of these findings for planning and design for nonstationary natural hazards are significant. Given a historic (or future) increasing trend in the magnitudes of a particular hazard, we should prepare to experience exceedance events much more frequently (Obeysekera and Salas, 2016). Through exploration of $S_{T}(t)$ for various trend factors, we also note that determining the reliability of a system over time is more complicated given uncertainty in the magnitude of the trend and how it will manifest. In either case, continuing to assume stationary conditions when computing system reliability for design purposes, when a positive trend in the POT magnitudes has been observed historically, may pose a 
significant risk to the populations and infrastructure in that region. Thus we recommend that design practices should be reviewed and adapted for cases where nonstationary behavior of natural hazards is evident in order to avoid under-design (also see Vogel et al., 2013).

Overall, we have shown that HFA provides a set of tools for understanding the probabilistic behavior of nonstationary natural hazards for application to a wide range of natural phenomena. We intend for this analysis to inform future work on modeling nonstationary natural hazards with HFA, for example by developing other models that may include co-variates (Villarini et al., 2013), extensions to AMS series, and also exploring the impact of decreasing trends. We expect that additional research on this topic will contribute to the emerging conversation on planning for nonstationary natural hazards and shed light on innovative methods to determine best practices for infrastructure design. Results of this work further support the need for a risk-based decision analysis framework for selecting a design event under nonstationarity (Rosner et al., 2014). Such a framework can provide guidance in choosing infrastructure that minimizes the risk of under-design (protection) and over-design (excess spending) through probabilistic decision analysis.

Acknowledgements. The authors would like to thank Jose D. Salas and one anonymous reviewer for their suggestions on this manuscript. This work was supported by the United States Army Corps of Engineers (USACE) Institute of Water Resources in part by an appointment to the USACE Research Participation Program administered by the Oak Ridge Institute for Science and Education (ORISE) through an interagency agreement between the US Department of Energy (DOE) and the USACE. ORISE is managed by ORAU under DOE contract number DE-AC0506OR23100. All opinions expressed in this paper are the authors' and do not necessarily reflect the policies and views of USACE, DOE or ORAU/ORISE. All data are freely available and analyzed with the R statistical software package version 3.1.1.

Edited by: K. Schröter

Reviewed by: J. D. Salas and one anonymous referee

\section{References}

Bayazit, M.: Nonstationarity of hydrological records and recent trends in trend analysis: a state-of-the-art review, Environ. Process., 2, 527-542, 2015.

Beguería, S., Angulo-Martínez, M., Vicente-Serrano, S. M., LópezMoreno, J. I., and El-Kenawy, A.: Assessing trends in extreme precipitation events intensity and magnitude using nonstationary peaks-over-threshold analysis: a case study in northeast Spain from 1930 to 2006, Int. J. Climatol., 31, 2102-2114, doi:10.1002/joc.2218, 2011.

Beirlant, J., Goegebeur, Y., Segers, J., and Teugels, J.: Statistics of Extremes: Theory and Applications, John Wiley and Sons, West Sussex, UK, 313-400, 2006.
Blaikie, P., Cannon, T., Davis, I., and Wisner, B.: At Risk: Natural Hazards, People's Vulnerability and Disasters, Routledge, London, UK, 1-50, 2014.

Bonnin, G. M., Maitaria, K., and Yekta, M.: Trends in Rainfall Exceedances in the Observed Record in Selected Areas of the United States, J. Am. Water Resour. Assess., 47, 1173-1182, 2011.

Coles, S., Bawa, J., Trenner, L., and Dorazio, P.: An Introduction to Statistical Modeling of Extreme Values, Springer, Bristol, UK, 17-47, 2001.

Cox, D. R.: Regression Models and Life-Tables, J. R. Stat. Soc. B., 34, 187-220, 1972.

Davison, A. C. and Smith, R. L.: Models for Exceedances over High Thresholds, J. R. Stat. Soc. Ser. B., 52, 393-442, 1990.

de Winter, R. C., Sterl, A., and Ruessink, B. G.: Wind extremes in the North Sea Basin under climate change: An ensemble study of 12 CMIP5 GCMs, J. Geophys. Res.-Atmos., 118, 1601-1612, doi:10.1002/jgrd.50147, 2013.

Di Baldassarre, G., Montanari, A., Lins, H., Koutsoyiannis, D., Brandimarte, L., and Blöschl, G.: Flood fatalities in Africa: From diagnosis to mitigation, Geophys. Res. Lett., 37, L22402, doi:10.1029/2010GL045467, 2010.

Ellsworth, W. L.: Injection-Induced Earthquakes, Science, 341, 1225942, doi:10.1126/science.1225942, 2013.

Fawcett, L. and Walshaw, D.: Sea-surge and wind speed extremes: optimal estimation strategies for planners and engineers, Stoch. Environ. Res. Risk A., 29, 1-18, doi:10.1007/s00477-015-1132$3,2015$.

Finkelstein, M.: Failure rate modelling for reliability and risk, Springer Science \& Business Media, Bloemfontein, South Africa, 9-65, 2008.

Guha-Sapir, D., Hargitt, D., and Hoyois, P.: Thirty Years of Natural Disasters 1974-2003: The Numbers, Presses univ. de Louvain, Belgium, 2004.

Gumbel, E. J.: Statistics of Extremes, Courier Corporation, Mineola, New York, 1-155, 2012.

Gutenberg, B. and Richter, C.: Seismicity of the earth and associated phenomena, Princeton, New Jersey, 241-256, 1954.

Hillier, F. S. and Lieberman, G. J.: Introduction to stochastic models in operations research, McGraw-Hill Companies, New York, 747-763, 1990.

Holmes, T. P., Huggett Jr., R. J., and Westerling, A. L.: Statistical Analysis of Large Wildfires, in: The Economics of Forest Disturbances, edited by: Holmes, T. P., Prestemon, J. P., and Abt, K. L., Springer Netherlands, Medford, Massachusetts, 59-77, 2008.

Hosking, J. R. M. and Wallis, J. R.: Parameter and Quantile Estimation for the Generalized Pareto Distribution, Technometrics, 29, 339-349, doi:10.1080/00401706.1987.10488243, 1987.

Jagger, T. H. and Elsner, J. B.: Climatology models for extreme hurricane winds near the United States, J. Climate, 19, 32203236, doi:10.1175/JCLI3913.1, 2006.

Katz, R. W. and Brown, B. G.: Extreme events in a changing climate: variability is more important than averages, Climatic Change, 21, 289-302, doi:10.1007/BF00139728, 1992.

Katz, R. W., Brush, G. S., and Parlange, M. B.: Statistics of Extremes: Modeling Ecological Disturbances, Ecology, 86, 11241134, doi:10.1890/04-0606, 2005.

Keellings, D. and Waylen, P.: Increased risk of heat waves in Florida: Characterizing changes in bivariate heat wave 
risk using extreme value analysis, Appl. Geogr., 46, 90-97, doi:10.1016/j.apgeog.2013.11.008, 2014.

Khaliq, M. N., Ouarda, T. B. M. J., Ondo, J.-C., Gachon, P., and Bobée, B.: Frequency analysis of a sequence of dependent and/or non-stationary hydro-meteorological observations: a review, J. Hydrol., 329, 534-552, doi:10.1016/j.jhydrol.2006.03.004, 2006.

Kiefer, N. M.: Economic Duration Data and Hazard Functions, J. Econ. Lit., 26, 646-679, 1988.

Kim, Y., Jang, S.-C., and Lim, T.-J.: Hazard analysis of typhoonrelated external events using extreme value theory, Nucl. Eng. Technol., 47, 59-65, doi:10.1016/j.net.2014.08.001, 2015.

Kleinbaum, D. G. and Klein, M.: Survival Analysis, Springer, New York, 1-114, 1996.

Klein, J. P. and Moeschberger, M. L.: Statistics for biology and health, Stat. Biol. Health, New York, 27-238, 1997.

Lansey, K. E., Duan, N., Mays, L. W., and Tung, Y. K.: Water distribution system design under uncertainties, J. Water Res. Pl.-ASCE, 115, 630-645, doi:10.1061/(ASCE)07339496(1989)115:5(630), 1989.

Lee, K. S., Sadeghipour, J., and Dracup, J. A.: An approach for frequency analysis of multiyear drought durations, Water Resour. Res., 22, 655-662, doi:10.1029/WR022i005p00655, 1986.

Liu, Y., Stanturf, J., and Goodrick, S.: Trends in global wildfire potential in a changing climate, Forest Ecol. Manage., 259, 685697, doi:10.1016/j.foreco.2009.09.002, 2010.

Lopatoukhin, L. J., Rozhkov, V. A., Ryabinin, V. E., Swail, V. R., Boukhanovsky, A. V., and Degtyarev, A. B.: Estimation of Extreme Wind Wave Heights, Secretariat of the World Meteorological Organization, Geneva, 1-73, 2000.

Madsen, H., Rasmussen, P. F., and Rosbjerg, D.: Comparison of annual maximum series and partial duration series methods for modeling extreme hydrologic events: 1. At-site modeling, Water Resour. Res., 33, 747-757, doi:10.1029/96WR03848, 1997.

Méndez, F. J., Menéndez, M., Luceño, A., and Losada, I. J.: Estimation of the long-term variability of extreme significant wave height using a time-dependent Peak Over Threshold (POT) model, J. Geophys. Res.-Oceans, 111, C07024, doi:10.1029/2005JC003344, 2006.

Obeysekera, J. and Park, J.: Scenario-Based Projection of Extreme Sea Levels, J. Coast. Res., 29, 1-7, doi:10.2112/JCOASTRESD-12-00127.1, 2012.

Obeysekera, J. and Salas, J. D.: Frequency of Recurrent Extremes under Nonstationarity, J. Hydrol. Eng., doi:10.1061/(ASCE)HE.1943-5584.0001339, in press, 2016.

Palutikof, J. P., Brabson, B. B., Lister, D. H., and Adcock, S. T.: A review of methods to calculate extreme wind speeds, Meteorol. Appl., 6, 119-132, doi:10.1017/S1350482799001103, 1999.

Pickands III, J.: Statistical inference using extreme order statistics, Ann. Stat., 3, 119-131, 1975.

Pike, M. C.: A Method of Analysis of a Certain Class of Experiments in Carcinogenesis, Biometrics, 22, 142-161, doi: $10.2307 / 2528221,1966$.

Pisarenko, V. F. and Sornette, D.: Characterization of the Frequency of Extreme Earthquake Events by the Generalized Pareto Distribution, Pure Appl. Geophys., 160, 2343-2364, doi:10.1007/s00024-003-2397-x, 2003.

Prosdocimi, I., Kjeldsen, T. R., and Svensson, C.: Non-stationarity in annual and seasonal series of peak flow and precipitation in the UK, Nat. Hazards Earth Syst. Sci., 14, 1125-1144, doi:10.5194/nhess-14-1125-2014, 2014.

Pryor, S. C. and Barthelmie, R. J.: Climate change impacts on wind energy: A review, Renew. Sustain. Energy Rev., 14, 430-437, doi:10.1016/j.rser.2009.07.028, 2010.

Read, L. K.: Planning and communicating risk for nonstationary natural hazards, PhD thesis, ProQuest Dissertations Publishing, Tufts University, USA, 6-140, 2016.

Read, L. K. and Vogel, R. M.: Reliability, return periods, and risk under nonstationarity, Water Resour. Res., 51, 6381-6398, doi:10.1002/2015WR017089, 2015.

Renard, B., Lang, M., and Bois, P.: Statistical analysis of extreme events in a non-stationary context via a Bayesian framework: case study with peak-over-threshold data, Stoch. Environ. Res. Risk A., 21, 97-112, doi:10.1007/s00477-006-0047-4, 2006.

Rosner, A., Vogel, R. M., and Kirshen, P. H.: A risk-based approach to flood management decisions in a nonstationary world, Water Resour. Res., 50, 1928-1942, doi:10.1002/2013WR014561, 2014.

Roth, M., Buishand, T. A., Jongbloed, G., Klein Tank, A. M. G., and Zanten, V. J.: A regional peaks-over-threshold model in a nonstationary climate, Water Resour. Res., 48, W11533, doi:10.1029/2012WR012214, 2012.

Roth, M., Buishand, T. A., Jongbloed, G., Klein Tank, A. M. G., and van Zanten, J. H.: Projections of precipitation extremes based on a regional, non-stationary peaks-over-threshold approach: A case study for the Netherlands and north-western Germany, Weather Clim. Extrem., 4, 1-10, doi:10.1016/j.wace.2014.01.001, 2014.

Ruggiero, P., Komar, P. D., and Allan, J. C.: Increasing wave heights and extreme value projections: The wave climate of the U.S. Pacific Northwest, Coast. Eng., 57, 539-552, doi:10.1016/j.coastaleng.2009.12.005, 2010.

Salas, J. D. and Obeysekera, J.: Revisiting the Concepts of Return Period and Risk for Nonstationary Hydrologic Extreme Events, J. Hydrol. Eng., 19, 554-568, doi:10.1061/(ASCE)HE.19435584.0000820, 2014.

Schoenberg, F. P., Peng, R., and Woods, J.: On the distribution of wildfire sizes, Environmetrics, 14, 583-592, doi:10.1002/env.605, 2003.

Smith, R. L.: Extreme Values in Finance, Telecommunications, and the Environment, CRC Press, Boca Raton, FL, 1-65, doi:10.1201/9780203483350.ch1, 2003.

Stedinger, J. R., Vogel, R. M., and Foufoula-Georgiou, E.: Frequency analysis of extreme events, in: Chapter 18 in Handbook of Hydrology, edited by: Maidment, D. R., McGraw-Hill, New York, 118-135, 1993.

Strupczewski, W. G., Singh, V. P., and Feluch, W.: Non-stationary approach to at-site flood frequency modelling I. Maximum likelihood estimation, J. Hydrol., 248, 123-142, doi:10.1016/S00221694(01)00397-3, 2001.

Sugahara, S., da Rocha, R. P., and Silveira, R.: Non-stationary frequency analysis of extreme daily rainfall in Sao Paulo, Brazil, Int. J. Climatol., 29, 1339-1349, doi:10.1002/joc.1760, 2009.

Thompson, E. M., Baise, L. G., and Vogel, R. M.: A global index earthquake approach to probabilistic assessment of extremes, J. Geophys. Res.-Sol. Ea., 112, B06314, doi:10.1029/2006JB004543, 2007.

Todorovic, P.: Stochastic models of floods, Water Resour. Res., 14, 345-356, doi:10.1029/WR014i002p00345, 1978. 
Tramblay, Y., Neppel, L., Carreau, J., and Najib, K.: Nonstationary frequency analysis of heavy rainfall events in southern France, Hydrolog. Sci. J., 58, 280-294, doi:10.1080/02626667.2012.754988, 2013.

Tung, Y.-K.: Models for Evaluating Flow Conveyance Reliability of Hydraulic Structures, Water Resour. Res., 21, 1463-1468, doi:10.1029/WR021i010p01463, 1985.

Tung, Y.-K. and Mays, L. W.: Risk models for flood levee design, Water Resour. Res., 17, 833-841, doi:10.1029/WR017i004p00833, 1981.

Utsu, T.: Representation and analysis of the earthquake size distribution: a historical review and some new approaches, in: Seismicity Patterns, their Statistical Significance and Physical Meaning, Birkhäuser Basel, New York, 509-535, 1999.

Villarini, G., Smith, J. A., Serinaldi, F., Ntelekos, A. A., and Schwarz, U.: Analyses of extreme flooding in Austria over the period 1951-2006, Int. J. Climatol., 32, 1178-1192, doi:10.1002/joc.2331, 2012.
Villarini, G., Smith, J. A., Vitolo, R., and Stephenson, D. B.: On the temporal clustering of U.S. floods and its relationship to climate teleconnection patterns, Int. J. Climatol, 33, 629-640, doi:10.1002/joc.3458, 2013.

Vogel, R. M., Yaindl, C., and Walter, M.: Nonstationarity: Flood Magnification and Recurrence Reduction Factors in the United States, J. Am. Water Resour. Assess., 47, 464-474, doi:10.1111/j.1752-1688.2011.00541.x, 2011.

Vogel, R. M., Rosner, A., and Kirshen, P. H.: Brief Communication: Likelihood of societal preparedness for global change: trend detection, Nat. Hazards Earth Syst. Sci., 13, 1773-1778, doi:10.5194/nhess-13-1773-2013, 2013.

Waylen, P. R.: Statistical analysis of freezing temperatures in Central and Southern Florida, J. Climatol., 8, 607-628, 1988.

Wienke, A.: Frailty Models in Survival Analysis, CRC Press, Boca Raton, 4-20, 2010.

Young, I. R., Zieger, S., and Babanin, A. V.: Global Trends in Wind Speed and Wave Height, Science, 332, 451-455, doi:10.1126/science.1197219, 2011. 